\title{
Differential experiences of discrimination among ethnoracially diverse persons experiencing mental illness and homelessness
}

\author{
Suzanne Zerger', Sarah Bacon', Simon Corneau², Anna Skosireva', Kwame McKenzie³, Susan Gapka', \\ Patricia O Campo ${ }^{1,4}$, Aseefa Sarang ${ }^{5}$ and Vicky Stergiopoulos ${ }^{1,6^{*}}$
}

\begin{abstract}
Background: This mixed methods study explored the characteristics of and experiences with perceived discrimination in an ethnically diverse urban sample of adults experiencing homelessness and mental illness.

Methods: Data were collected in Toronto, Ontario, as part of a 4-year national randomized field trial of the Housing First treatment model. Rates of perceived discrimination were captured from survey questions regarding perceived discrimination among 231 ethnoracially diverse participants with moderate mental health needs. The qualitative component included thirty six in-depth interviews which explored how individuals who bear these multiple identities of oppression navigate stigma and discrimination, and what affects their capacity to do so.

Results: Quantitative analysis revealed very high rates of perceived discrimination related to: homelessness/poverty (61.5\%), race/ethnicity/skin colour (50.6\%) and mental illness/substance use (43.7\%). Immigrants and those who had been homeless three or more years reported higher perceived discrimination on all three domains. Analysis of qualitative interviews revealed three common themes related to navigating these experiences of discrimination among participants: 1) social distancing; 2) old and new labels/identities; and, 3) homeland cultures.

Conclusions: These study findings underscore poverty and homelessness as major sources of perceived discrimination, and expose underlying complexities in the navigation of multiple identities in responding to stigma and discrimination.
\end{abstract}

Trial registration: Current Controlled Trials ISRCTN42520374. Registered 18 August 2009.

\section{Background}

Toronto is home to the largest cohort of homeless people in Canada, with over 5,000 people literally homeless on any given night and approximately 28,000 different individuals using homeless shelters each year [1]. Toronto is also one of the worlds most multicultural centres, with half of its population born outside of Canada, and nearly as many half $(49 \%)$ describing themselves as belonging to a visible minority [2]. Newcomers represent twothirds of the population growth in Canada over the past decade, and it is projected that by $2031,78 \%$ of persons

\footnotetext{
* Correspondence: StergiopoulosV@smh.ca

'Centre for Research on Inner City Health, Li KaShing Knowledge Institute of St. Michael s Hospital, 209 Victoria Street, Toronto, Ontario M5C 1N8, Canada ${ }^{6}$ Department of Psychiatry, University of Toronto, 250 College Street, Toronto, Ontario M5T 1R8, Canada

Full list of author information is available at the end of the article
}

living in Torontos Census Metropolitan Area will be immigrants or Canadian-born children of immigrants [3]. Visible minorities, immigrants and refugees in Canada are especially vulnerable to becoming homeless [4-7] and homelessness, in turn, is a cause and consequence of complex health, mental health, and addiction problems $[8,9]$.

Experiences with stigma and discrimination are distressingly common for individuals who are visible minorities, homeless, and/or have a mental illness [10-16]. Negative consequences of stigma and discrimination are well-documented, particularly negative physical and mental health outcomes, the latter at least in part due to delay or avoidance of treatment [11-13,17-27]. Increasingly, researchers have taken into account the effects of multiple domains on discrimination experiences, such as mental illness, race, gender, age, physical disability, and 
sexual orientation. They have typically concluded that compound effects result in even worse health outcomes, and that all of these domains are important contributors when looking for effective interventions or, as one author put it, the capacity of the individual to resolve the negative impacts of discrimination [28] (see also: [15,29-34]). Yet while acknowledging that discrimination can be based on multiple domains of difference, the literature largely assumes an additive, not an intersectional, effect.

Most of these primarily US-based studies also lack multicultural samples and employ quantitative measures of discrimination prevalence. And while income and neighborhood poverty are occasionally included as potential factors affecting perceived discrimation, homelessness and immigration status are rarely considered.

In part because immigrants tend to experience housing instability in the form of residential crowding - sometimes called hidden homelessness - literature exploring their experiences with literal homelessness is scant $[35,36]$. However, some recent studies from Canada have assessed the physical and mental health of homeless immigrant populations [11,37-40], and a few qualitative studies have explored the experiences of homeless women, youth and families, including immigrants [41-44]. One Ontario qualitative study looked at factors affecting discrimination among homeless mothers with mental illness, and noted but did not pursue the finding that issues newcomers faced tended to compound the barriers for these women [42] p.691. A more recent Canadian study asserted the need for a better understanding of how discrimination and its health effects are differentially experienced by immigrants and refugees [45], p.1557. Authors of a review of discrimination and stigma measures related to mental illness note a gap in understanding how non-Western cultures experience mental illness stigma [46]. On the whole, much remains unknown about the relative prevalence of discrimination due to homelessness and immigration status, the factors associated with that prevalence, and especially about how multiple identities interact and inform experiences of stigma and discrimination.

This mixed method study takes advantage of a unique study sample with an ethnically diverse group of individuals who are experiencing homelessness and mental health problems to address some of the gaps in the literature. A key purpose of this study is to explore how individuals who bear multiple labels and identities of oppression including being homeless, an immigrant, a racialized minority, and having a mental illness, navigate stigma and discrimination and what affects their capacity to do so. Specifically, this study addresses three research questions: 1) What is the prevalence of perceived discrimination due to homelessness/poverty, race/ethnicity, and mental health/substance use in an ethnically diverse sample of homeless people with mental illness? 2) What sociodemographic characteristics are associated with perceived discrimination? And, 3) How do multiple identity characteristics interact and inform experiences of discrimination?

\section{Methods}

Given the lack of quantitative data on perceived discrimination based on poverty and homelessness, and our interest in qualitatively exploring the interplay between identities in multiple social locations simultaneously, we determined that a mixed methods approach would be most appropriate to address our research questions. This study uses data collected in Toronto as part of a 4-year national randomized field trial of the Housing First model for persons experiencing homelessness and mental illness. Details about this national trial - At Home/Chez Soi have been published elsewhere: $[47,48]$.

\section{Study sample}

In Toronto, At Home/Chez Soi participants were recruited from its extensive network of organizations serving people experiencing homelessness, including shelters, drop-in centres, community health centres, hospitals and criminal justice programs. Eligible participants were 18 years or older, absolutely homeless or precariously housed ${ }^{\mathrm{a}}$, had a severe mental illness with or without a co-occurring substance use problem, and were not currently receiving assertive community treatment or intensive case management. Individuals not meeting these criteria were ineligible for the study, as were those who were not a Canadian citizen, permanent resident, refugee or refugee claimant at the time of enrollment. Targeted recruitment strategies, published elsewhere [47], ensured the final sample was representative of the ethno-racial diversity of Toronto and characteristic of its homeless population. From the total sample of 550 participants in Toronto, for the purposes of this study we examined the subsample of 231 who identified as racialized minorities and rated as moderate need for mental health services $^{\mathrm{b}}$. For the qualitative component we used purposeful sampling methods; field coordinators identified 36 individuals who were most willing and able to reflect on their experiences. All of those identified agreed to participate in an in-depth interview.

\section{Sample description}

Three-quarters of the qualitative subsample $(n=36)$ interviewed were male and just over half (55\%) were younger than 40 years. Two-thirds had completed at least high school. One-third were born in Canada; the remainder were born in 19 different countries (see Table 1 footnote). Two-fifths had spent three or more years living homeless. Just over one-third were diagnosed 
Table 1 Selected demographic characteristics

\begin{tabular}{|c|c|c|c|c|}
\hline & \multicolumn{2}{|c|}{$\begin{array}{l}\text { Qualitative interview } \\
\text { participants }(\mathrm{N}=36)\end{array}$} & \multicolumn{2}{|c|}{$\begin{array}{l}\text { Ethno-racial moderate needs } \\
\text { participants }(\mathrm{N}=231)\end{array}$} \\
\hline & $\mathrm{n}$ & $\% *$ & $n$ & $\%^{*}$ \\
\hline \multicolumn{5}{|l|}{ Age } \\
\hline$<30$ years & 12 & 33.3 & 66 & 28.6 \\
\hline 30-39 years & 8 & 22.2 & 53 & 22.9 \\
\hline$\geq 40$ years & 16 & 44.4 & 112 & 48.4 \\
\hline \multicolumn{5}{|l|}{ Gender } \\
\hline Female & 9 & 25.0 & 75 & 32.5 \\
\hline Male & 27 & 75.0 & 152 & 65.8 \\
\hline Transgender & 0 & 0.00 & 4 & 1.7 \\
\hline \multicolumn{5}{|l|}{ Education } \\
\hline Less than high school & 12 & 33.3 & 104 & 45.0 \\
\hline Completed high school & 8 & 22.2 & 43 & 18.6 \\
\hline Some college/university & 16 & 44.4 & 84 & 36.4 \\
\hline \multicolumn{5}{|l|}{ Country of birth } \\
\hline Canada-born & 12 & 33.3 & 65 & 28.1 \\
\hline Foreign-born & $24^{* *}$ & 66.7 & 166 & 71.9 \\
\hline \multicolumn{5}{|l|}{ Homelessness: Lifetime duration } \\
\hline$<3$ years & 20 & 55.6 & 123 & 56.2 \\
\hline$\geq 3$ years & 16 & 44.4 & 96 & 43.8 \\
\hline \multicolumn{5}{|l|}{ Mental health: MINI results } \\
\hline Current psychotic disorder & 13 & 36.1 & 81 & 35.1 \\
\hline
\end{tabular}

*Percentages are based on valid responses. **Countries of birth in this group included: Canada (12), Jamaica (3), Afghanistan (2), Somalia (2), Uganda (2), Bangladesh, Barbados, Congo, Egypt, Ethiopia, Ghana, Grenada, Guyana, Kenya, India, Iran, Mexico, Sri Lanka, Trinidad and Tobago, and Zambia (each, $\mathrm{n}=1$ ).

with a psychotic disorder. These demographic characteristics were similar to the larger sample $(\mathrm{n}=231)$, though the latter included slightly fewer males (66\% compared to $75 \%$ ) and lower educational achievement overall (55\% completed high school or more, compared with $66 \%$ in the subsample) (Table 1).

This study was approved by the Research Ethics Board (REB) at St. Michael s Hospital, Toronto. At Home Study participants were recruited between October 2009 and June 2010; they provided written informed consent and were compensated for their participation. Separate REB approval was obtained for the qualitative interviews; participants signed a separate consent form and received additional compensation of $\$ 25$ cash and transportation costs for in-person interviews conducted between November 2010 and July 2011.

\section{Data sources}

\section{Quantitative}

We use findings from discrimination questions included in the baseline interviews with the sample of 231 ethnoracial moderate needs participants. Specifically, participants were asked whether they had experienced discrimination or been treated unfairly by others in Canada due to: ethnicity, race, skin colour, language, accent, religion, gender, sexual orientation, appearance, mental health, use of alcohol or drugs, homelessness, or poverty. We grouped participants according to whether they perceived experiencing discrimination due to (i) homelessness or poverty, (ii) mental health or use of alcohol or drugs, and (iii) race or ethnicity or skin colour. Selected demographic variables, adapted from the 2006 Census of Canada, were also collected and used to describe these two samples, and the Mini International Neuropsychiatric Interview 6.0 (MINI 6.0) was used to assess and describe mental health and substance use disorders [48].

\section{Qualitative}

A trained researcher conducted in-person interviews, lasting between 34 and 90 minutes, with the subsample of 36 ethnoracial moderate needs participants. The semistructured interview protocol was designed to probe for experiences with intersecting dimensions of homelessness, mental illness, race and gender or any other possible forms of discrimination or stigma; for example, after general questions about discrimination experiences with homelessness, participants were asked to reflect whether/ how their mental health status, ethnicity, or gender may 
have influenced those experiences. Though asked generally about being treated unfairly, the concepts of discrimination and stigma were not explicitly defined, as the intent was to better understand how the interviewees conceived of and described experiences associated with these identities. Though interpreters were available, all chose to conduct their interviews in English. All interviews were audio-taped and transcribed.

\section{Analytic strategies \\ Quantitative}

SPSS 20.0 software was used for the quantitative analysis. Descriptive statistics, including frequencies and means, were used to describe both study samples. Crosstabulations and Pearson Chi Square tests and Fishers Exact Test for cell sizes fewer than 4 were used to assess statistically significant differences of categorical demographic variables on perceived discrimination.

\section{Qualitative}

We used data analytical techniques of grounded theory to analyze transcribed interview text. Specifically, we used coding and comparative analytic inductive procedures to de-contextualize and re-contextualize individual cases and experiences [49]. This iterative process led to increasingly abstract conceptual categories which helped to expose the data and patterns within it [50]. The research analyst first conducted line-by-line open coding during a thorough read of the transcripts to identify key concepts, then progressively transformed codes into higher level categories by using a constant comparative method of analyzing and grouping similar codes into conceptual categories ( axial coding). A constructionist stance guided the process, which meant the focus was to identify latent thematic schema, as opposed to semantic or descriptive [51]. A team of qualitative analysts compared several coded transcripts to ensure consistency at regular intervals throughout this process. NVivo 9.2 software facilitated the coding and thematic analysis process, and memo notes were used to track emerging codes. To enhance rigor, the researcher kept a journal to track and address potential assumptions or biases, and listened to audio-tapes after coding to gain contextual insight that may have been missed in the transcribed version.

\section{Results}

The Quantitative section describes characteristics of perceived discrimination among our sample of 231 homeless, ethnoracially diverse moderate needs participants, while the Qualitative section synthesizes findings from the analysis of interviews with the subsample of 36 participants.

\section{Quantitative}

\section{Perceived discrimination findings}

Perceived discrimination among study participants is very high, with $61.5 \%$ reporting perceived discrimination due to homelessness or poverty, $50.6 \%$ due to race, ethnicity, or skin colour, and $43.7 \%$ due to mental health problems or alcohol/drug use. Nearly three-quarters of the sample $(73.6 \%)$ reported perceived discrimination on at least one of these domains (Table 2).

Univariate analysis showed that age, gender, education, employment status and the diagnosis of psychotic disorder were not related to any of the three domains of perceived discrimination. (See Additional file 1) Individuals who experienced discrimination in any of the three domains showed statistically significant difference in terms of their ethnic or cultural identity: more than half of those who experienced each form of discrimination identified their ethnicity as Black. Individuals who reported perceived discrimination due to homelessness or poverty and due to mental health or alcohol/drug problems had significantly lower monthly income compared to those who did not report any of these types of discrimination. Individuals born outside of Canada were more likely to report perceived discrimination compared to their Canadian-born counterparts, and this pattern was significant across all three forms of discrimination. Individuals who experienced discrimination of any of the three forms were more likely to have been homeless for three or more years $(x 2(1, N=170)=8.80)$. We obtained similar results (not reported) by testing each form of perceived discrimination against the group which did not experience any type of discrimination.

\section{Qualitative}

\section{A note on perceived discrimination experiences}

All of those interviewed described being stigmatized due to their differences, ranging from appearance (traditional ethnic dress, unkempt clothing due to living on the street, skin colour), to behaviors (typically associated with mental illness or substance use). And most had encountered discrimination, primarily when seeking jobs or housing, or interacting with law enforcement or service providers.

Table 2 Prevalence of perceived experiences of discrimination in any setting during Prior 12-months, Toronto at Home/Chez Soi Ethno-Racial moderate needs participants

\begin{tabular}{lll}
\hline & \multicolumn{1}{c}{$(\mathbf{n}=\mathbf{2 3 1 )}$} \\
\cline { 2 - 3 } Perceived discrimination due to & $\mathbf{n}$ & \% \\
\hline Homelessness or poverty & 142 & 61.5 \\
Ethnicity, Race, or Skin colour & 117 & 50.6 \\
Mental health or alcohol/drug use & 101 & 43.7 \\
Any discrimination & 170 & 73.6 \\
\hline
\end{tabular}


Participants subgroup identities are inextricably linked as they intersect with perceptions of, and experiences with, stigma and discrimination. These individuals certainly do not perceive themselves nor experience discrimination categorically: as one woman commented, I would get put down because not only am I brown but I $\mathrm{m}$ a woman that s out on the streets. However, this qualitative component is focused not on documenting or describing these experiences, but rather on uncovering how their multiple interlocking identities affect these experiences. A companion article from the At Home/Chez Soi study, published elsewhere, explored the perceptions of discrimination in healthcare settings among this populaion [52].

\section{Theme: Social distancing}

A recurrent theme in participant narratives described social distancing, the reasons underlying it, and its impact on coping and recovery.

Reasons for social distancing Interviewees described distancing themselves from family and friends because of the stigma associated with their experiences of homelessness and/or mental illness. One man said homelessness made him feel worthless: I stopped trusting anyone. I $\mathrm{m}$ isolating myself; another discussed avoiding contact to hide his mental illness: I tried to be isolated from anyone, so no one can know that I m sick.

Nearly all of those who immigrated as adults additionally described distancing themselves from family and friends in their home country, declaring they had not informed them about their homelessness or, if diagnosed in Canada, their mental illness. They spoke about the shame of being homeless or mentally ill, of feelings of failure to meet social norms and expectations and not wanting to disappoint worry or disturb their family. Typical comments included: I don t want my mom to get depressed and worry about me; and I just didn $t$ want to have to put them through that.

Many Canadian-born respondents also talked about social distancing, not trusting others, and not wanting to cause worry or be a burden; however, their families and friends were more apt to know about and in some cases had played a direct role in - their pathways to homelessness and/or mental health problems. Several noted that their family members were also poor and busy trying to survive. Some avoided family members or friends who were substance abusers or triggers for their mental health problems as a means to aid in their own recovery.

Female interviewees were more apt to talk about social distancing as a survival technique. As this woman explained: I don $t$ get myself involved in any situation I can t come out of I stay by myself.
Varied effects of social distancing Even when social distancing was not a direct result of stigma, and even when it was deemed necessary for recovery to progress, it had myriad negative effects which entrenched participants further into poverty and homelessness. For example, it cut off important sources of emotional or instrumental support, created and/or exacerbated depression or other mental health problems, and reinforced a lack of trust in others, thus hindering engagement with social services. All of them understood and felt the negative consequences; one articulated it this way: I really kept myself isolated most of the time. So by doing so I lost contacts, I lost maybe job opportunities, maybe some encouragement which maybe I could have got from friends.

Among foreign-born interviewees, the profound sense of being alone reinforced their helplessness about finding a way out of their predicaments. As one man commented: What else can you do. You are here in a foreign land. You cant run to your father to your sister to your mother to talk about it. The only person maybe is a caseworker, who gives you an appointment. Yet while distancing and self-isolation impeded access to critical supports in Canada, it also served as a means to protect and retain such support from family/friends abroad. Many described their family as key to their survival: for example, when I remember them I find there is something in my life I can live for. By not revealing their experiences of homelessness and/or mental illness to those most important to them, they were able to project a more positive self-image, prevent additional stigma, and retain emotional support. This recent immigrant explained why he distanced himself from his family: I didn $\mathrm{t}$ want to scare them, because I was their strength. My children, and my wife, they knew I was their hero.

Summary Discrimination and stigma associated with being homeless or having a mental illness, led all of these participants to distance themselves from family and friends, and experience profound loneliness and loss of critical supports. The motives and effects of social distancing, however, varied within and among subgroups: for example, women tended to talk about it more as a tool of survival than as a means of avoiding shame, and recent immigrants distanced themselves from family and friends in their homelands to protect loved ones and retain meaningful emotional supports.

\section{Theme: Old and new labels}

Another recurring theme was the qualitatively different ways that those interviewed described experiences with the three kinds of discrimination we asked about: race/ ethnicity, mental illness, and homelessness. Being a person of colour is an old label : race discrimination was something participants were familiar with; they had 
grown up experiencing and learning to ignore or otherwise cope with that is, racism was nothing new. Acquiring a diagnosis of a mental illness or becoming homeless, on the other hand, were mostly new labels and identities which required new and pressing internal negotiation to determine how it fit or did not fit with who they are, and thus how they would respond to stigma or discrimination based on them. Though this theme held for all participants, there were again differences between foreign- and Canadian-born participants.

Race/Ethnicity/Skin colour: Old labels Asked directly about any experiences they might have with discrimination based on their colour or race, most of the immigrants in this sample said they had not experienced it at all since arriving in their new country. These responses were frequently tied to their perceptions of Canada and the multicultural context of Toronto, as illustrated by the following comments: I heard from my friend ..[that] there is no discrimination here; and, I never got any racial discrimination that I knew of . These perfunctory assertions, though, belie the reality that they were simply accustomed to race discrimination in its many forms and had become inured to its inevitability. Their comments included, for example: my upbringing was already training me to deal with racism; It doesn t bother me because I grew up with it, I know how to deal with it; and, it used to bother me, but after you see something repetitive after like, after five, ten years, you re like, yeah, whatever.

While Canadian-born participants were more apt to mention specific experiences of race discrimination, a few talked about it as becoming more insidious. As one woman put it, its changing forms now its something more sneaky. Yet they too were much more apt to focus on stigma associated with mental illness or homelessness.

Homelessness and mental illness: New labels Most expressed reactions to being diagnosed with a mental illness and/or becoming homeless in dramatic, lifealtering terms: I was kind of shocked, it was just this overwhelming feeling; oh, it ruined my whole life ; and, mental health kind of just snatched me out of my life. Several initially reacted with denial: I didn t believe it I said I m normal, I just had some stability issues ; and, I couldn $\mathrm{t}$ believe that I was sick. Similarly, many especially foreign-born participants described becoming homeless as completely new and confusing: it was totally new, totally new; and it was something all of a sudden and something that I ve never heard about.

For many respondents, becoming homeless was inextricably linked to their mental illness. One man described his diagnosis as the reason he was put on the streets; another noted his was another thing I would have avoided if I wasn $t$ homeless I wouldn t have to have an assessment for my mental health.

That they more frequently verbalized stigma and discrimination from homelessness than from mental illness may stem in part from homelessness being more visible, as they could more easily hide their mental illness from others while getting accustomed to treatment and others reactions: not many people have asked me, so no, I haven $t$ been discriminated on the basis of mental illness; and, If I didn t tell you that I had ADHD and bipolar, you wouldn $t$ have known, right?

Asked about experiences with being stigmatized due to these new label identities, they primarily talked about the process of learning what these new labels had to do with who they were: it was puzzling at first because I didn t expect people to be acting that way; and, Its not you who says that you are homeless. People they define you as homeless. The first time he encountered a homeless shelter, one immigrant man said: I didn $t$ get used to the adjustment very well. I didn $t$ deal with it I was trying to pretend that I wasn there. Another commented: It just feels like I m walking but I don $t$ feel myself I have really heavy bags to carry and I know that I exist but then I don t feel myself kind of half semi-conscious.

Summary Interviewees described experiences with discrimination associated with race, homelessness, and mental illness, in qualitatively different ways. Adjusting to race discrimination was not new, but navigating stigma associated with mental illness diagnoses and homelessness were new and unsettling experiences. For most, becoming homeless and/or diagnosed with a mental illness involved an overwhelming sense of failure, loneliness, and sadness, and many blamed themselves, thus internalizing the stigma they experienced from others. Participants frequently linked becoming homeless with being diagnosed with a mental illness, but found it easier to mask and avoid the stigma associated with mental illness.

\section{Theme: Homeland cultures}

Another factor that influenced how these individuals processed new label identities - homeless and mentally ill and associated stigmas, was how these were perceived in their culture and/or birth country. Though it tended to affect recent immigrants more strongly, especially those with families still in their homeland, it also affected Canadian-born respondents with relatives or friends who were still closely tied to their homeland and its culture.

Mental illness Reactions to stigma associated with mental health diagnoses were shaped by perceptions of mental 
illness in the culture of their home country. The words they used to describe how mental illness was perceived (e.g. outcasts and crazy dangerous and someone screaming and beating people ) can be read as a desire to contrast those perceptions with their personal experiences. One man said in his home country mental illness meant that you re a crazy person. I couldn $t$ believe I was sick because I m belonging now to crazy people.

Those who struggled the most to come to terms with a medicalized, diagnostic understanding of their mental health symptoms and issues in Canada tended to be those from cultures with non-medicalized concepts of mental illness. For example, they tend to think, like, you re bewitched and think a spell was put on you. They don $t$ take it like it might just be a medical thing. Many responded to the cultural dissonance by further distancing themselves from and even blaming - those individuals and cultural organizations which had previously been important sources of support. For example, one man who had immigrated to Canada as a boy blamed his inability to communicate with his parents about his experiences with depression (and treatment) on his cultural roots: They don $t$ understand what s a mental illness. Another man avoided taking medications to control symptoms because his priest suggested they came from a Satanic source he thought I had evil spirit inside me. Though he took communion as advised, his condition worsened; he subsequently avoided church, which had been an important source of support for him. A young woman raised primarily in Canada said her otherwise supportive parents kind of knew she had mental issues but did not seek psychiatric help, and did not believe in medications, because, [back home] they think of it in spiritual terms, not medical so I think culture does play a huge role.

Homelessness Similarly, many respondents used very strong language to describe how people from their homeland/culture perceived homeless people: like a dog that doesn $t$ have an owner No home, no hope; people really don t talk or sit with that person; you can die in the street, just like an animal; and, someone who doesn $t$ deserve help.

This perception clashed with their personal experiences: [People back home would say] you came to the west its a land of opportunity, so you should be able to better yourself but its actually hard to make money. Another said he had assumed homelessness was something that happened to orphans or those with difficult family relations not to someone from a decent family like his; he therefore struggled when others would look at you like its your fault that you re homeless.

Though negative stereotypes of homelessness were mostly consistent across all respondents, there were some distinctions by country of origin. One man from Colombia, for example, described homelessness as a symptom of an impoverished country where a war is going on and people who are living on the street are treated as people that need help. A few interviewees from Jamaica noted the importance of family members in preventing homelessness: in our culture you take your family in.

Summary Cultural perspectives and beliefs played an important role in how these individuals interpreted and adjusted to their newfound statuses as mentally ill and/or homeless and the associated stigma and discrimination they experienced. Many coming to terms with a medicalized understanding of mental illness and the reality of living homeless reacted to cultural dissonance by distancing themselves from their cultural origins.

\section{Discussion}

Perceived discrimination was very common among this ethnically diverse urban sample of 231 homeless individuals with mental illness, especially perceived discrimination due to homelessness and poverty. Those who had spent three or more years without stable housing in their lifetime, and those who were foreign born, were especially likely to report discrimination due to homelessness/poverty, to mental health/substance use, and to race/ethnicity/skin color when compared with those with less experience living homeless and Canadian-born individuals.

As noted previously, literature on the prevalence of discrimination due to homelessness and poverty is scarce, especially in samples of homeless adults, so it is difficult to say how these findings compare to other samples. One study, for example, found $53 \%$ of a sample of 1,824 individuals with severe mental illness experienced some form of discrimination; this included 51.5\% due to economic circumstance and $21.5 \%$ due to homelessness [30]. About a third of a sample of 500 Black and 419 Latino active substance users in another study reported discrimination due to poverty (38\% of Black; $26 \%$ Latinos) [53]. Finally, about one-third of 523 individuals living with HIV-AIDS in temporary housing reported experiencing discrimination due to homelessness, but was specific to health-care settings [54]. While these examples suggest our finding of $61.5 \%$ experiencing perceived discrimination due to homelessness or poverty is high, the samples described above are all US-based with limited racial diversity, and are not directly comparable.

Our findings led us to explore, with a subgroup of 36 participants, factors which affected how they perceived and experienced stigma or discrimination associated with their race, homelessness, or mental illness, and to focus more closely on immigrants in our sample. We 
identified three overarching themes: social distancing; old and new labels; and homeland cultures. Addressing multiple identities allowed for an exploration of the complexity of these participants experiences, and helped identify the unique effects of culture and newcomer status.

All of these participants distanced themselves from friends and family due to shame and the stigma of being homeless and/or diagnosed with a mental illness. Aspects of this theme recur in the literature: social-isolation can be both a consequence and a survival mechanism for individuals experiencing homelessness [55,56], and stigma associated with mental illness is shown to undermine social connectedness [57]. In the present study, those born outside of Canada tended to consider their family back home as essential to their survival yet were least apt to communicate with them about their struggles and despair. The physical distance, and having control over when/how communication occurred, meant that even if they could not give financial support, they could at least protect loved ones from worrying, and maintain aspects of much needed positive self image. This finding suggests the importance of understanding social distancing as a nuanced reaction which may vary for subgroups.

Study participants spoke very differently about discrimination due to race than discrimination due to homelessness and mental illness. The latter were new labels which required them to internally negotiate what these identities meant for them, and were most disarming. The stigma of being homeless roused especially deep shame; unlike race, homelessness is situational and subject to at least some potential for change, and unlike mental illness, can be hard to hide from others. The role and impact of each of these labels/identities is discussed in the literature, but this study suggests they can have very different meanings and roles depending on social context, and should not be analyzed in isolation [58].

Finally, the perceptions of mental illness and homelessness in participants homeland or culture affected how they interpreted and adjusted to these new labels. Some research has tied cultural belief systems to mental health, though it remains predominantly focused on firstworld countries [59]. Our study found participants from cultures that do not medicalize mental illness were less likely to seek/find empathy from family/friends both at home or within their local ethnic communities. Similarly, a recent study found in some countries which lacked mental health services there was a lack of a perceived norm about mental health, and this served as a barrier to service access for some first-generation U.S. immigrants [60]. The present study, though, found that discrimination experiences were especially distinct for those participants most closely tied to a culture or homeland outside of Canada, regardless of their immigration status or length of time residing in Canada. That is, this study suggests cultural ties play a larger role than birthplace in shaping experience of discrimination, and would be an important factor in a more systematic investigation.

Interest in examining discrimination along multiple domains is growing, yet remains limited to two or three social categories; more diverse study samples and alternative frameworks, such as intersectionality, should be applied to ensure a comprehensive understanding of how social classifications mutually depend on each other for meaning and, thus, mutually construct one another and work to shape outcomes [61]. As illustrated with this study, the processes with which multiple identities interact in shaping how stigma and discrimination are experienced and negotiated are nuanced and complex. A description of one of the participants situations illustrates this: This man immigrated to Canada as a boy, sponsored by his uncle. When his uncle died from a heart attack a few years later, he had no one to guide him and became homeless. When I became homeless it had an effect on my mental health I was very depressed, I was sad all the time, I was hopeless. Thats why I became suicidal once. So they took me to the psychiatric hospital. His depression led him to stop talking to his family and friends ( $\mathrm{I}$ isolate from everyone ). He felt shame from the stigma about his homelessness and believed his family - living abroad would not understand his homelessness or his mental illness. His homelessness, his race, his immigrant status, and his depression when combined with social distancing, discrimination, stigma and misfortune - all hindered his capacity to exit homelessness. To neglect any of his social categories/identities, or even to focus on one more than on others, would be to lose critical meaning of his experience and, therefore, an understanding about how best to support him. As one author noted, to focus on a single dimension in the service of parsimony is a kind of false economy [61], p.179. As importantly, shifting from a traditional additive approach e.g. an individual with two minority statuses experiences more discrimination than someone with one reveals the complex processes in which social categories and locations shape discrimination experiences and individuals reactions to them.

\section{Study limitations}

This studys limitations included: 1) Participants in this study all reside in Toronto, Ontario. While the uniquely multi-ethnic context made it possible to recruit the diverse sample for this study, it may have ameliorated effects of some of the racial discrimination these participants experienced. 2) Purposive sampling methodology meant restricting the sample to those willing and able to reflect on their experiences. And, although interpreters were available, all respondents opted for interviews in 
English [this also occurred in a qualitative study with homeless mothers [42]]. We thus did not explore discrimination due to language as distinct from race/ethnicity, although it did not arise even among study participants with limited English proficiency. 3) Experiences with discrimination and stigma were based on self-reports and perceptions, so it is not possible to verify specific examples given; however, perceived discrimination itself has been linked to detrimental outcomes [12].

\section{Conclusions}

This study revealed very high rates of perceived discrimination among ethnoracially diverse persons experiencing homelessness and mental illness, and exposed underlying complexities in the navigation of multiple identities in responding to stigma and discrimination, particularly for immigrants and others closely tied to countries of origin.

Becoming literally homeless, and/or of being newly diagnosed with a mental illness, had a profoundly devastating impact on the lives of study participants. Future research should not only heed the enormity of these causes for discrimination, and explore key elements in service delivery that might support coping and resilience, but also strive to disentangle the complex effects these identities have on various subgroups; as this research revealed, for example, reactions traditionally seen as negative (such as social distancing) can have important positive consequences for women and for individuals with social support systems living abroad.

\section{Endnotes}

${ }^{a}$ Absolutely homeless: no fixed place to stay for at least the past 7 nights with little likelihood of finding a place in the upcoming month. Precariously housed: housed in single room occupancy (SRO), rooming house, or hotel/ motel as a primary residence AND in the past year have a history of 2 or more episodes of being Absolutely Homeless OR one episode of being absolutely homeless of at least 4 weeks duration in the past year.

${ }^{\mathrm{b}}$ Criteria for establishing moderate need was based on an algorithm which considered community functioning, mental disorder diagnosis, co-morbid substance use, prior hospitalizations and incarcerations, and results from the Multnomah Community Ability Scale (MCAS). See additional detail in 48 .

\section{Additional file}

Additional file 1: Table S1. Chi-square tests comparing individuals with and without perceived experiences of discrimination during prior 12-months on selected socio-demographic characteristics and presence of psychosis, Toronto At Home/Chez Soi Ethno-Racial Moderate Needs Participants $(n=231)$
Competing interests

The authors declare that they have no competing interests.

\section{Authors contributions}

VS and SC conceived of and designed the study (VS additionally coordinated the study); SB and SZ conducted analyses and drafted the manuscript; AS conducted statistical analyses; KK, SG, PO and AS made substantial contributions to the analysis and interpretation of data. All authors read and approved the final manuscript.

\section{Acknowledgements}

We thank Jayne Barker (2008-11), Ph.D., Cameron Keller (2011-12), and Catharine Hume (2012-present) Mental Health Commission of Canada At Home/Chez Soi National Project Leads, Paula Goering, Ph.D. The National Research Lead, the National Research Team, the five site research teams, the Site Co-ordinators, and the numerous service and housing providers, as well as persons with lived experience, who have contributed to this project and the research. This research has been made possible through a financial contribution from Health Canada. The views expressed herein solely represent the authors.

\section{Author details}

${ }^{1}$ Centre for Research on Inner City Health, Li KaShing Knowledge Institute of St. Michael s Hospital, 209 Victoria Street, Toronto, Ontario M5C 1N8, Canada. ¿Universit du Qubec Montral, 405 Rue Sainte-Catherine Est, Montral,

Qubec, H2L 2C4, Canada. ${ }^{3}$ Health Services and Health Equity Research, Centre for Addiction and Mental Health, 250 College Street, Toronto, ON M5T 1R8, Canada. ${ }^{4}$ Dalla Lana School of Public Health, University of Toronto, 55 College St, Toronto, ON M5T 3M7, Canada. ${ }^{5}$ Across Boundaries: An Ethno-racial Mental Health Centre, 51 Clarkson Ave, Toronto (Ontario), M6E 2T5, Canada. ${ }^{6}$ Department of Psychiatry, University of Toronto, 250 College Street, Toronto, Ontario M5T 1R8, Canada.

Received: 25 June 2014 Accepted: 5 December 2014

Published online: 14 December 2014

\section{References}

1. City of Toronto: Total number of unique individuals using the shelter system 2000-2008. 2009; www.toronto.ca/legdocs/mmis/2009/cd/bgrd/ backgroundfile-24924.pdf. Accessed December, 2014.

2. City of Toronto. 2011 National Household Survey: Immigration, Citizenship, Place of Birth, Ethnicity, Visible Minorities, Religion and Aboriginal Peoples. Backgrounder. http://www1.toronto.ca/city_of_toronto/social_development_ finance_administration/files/pdf/nhs_backgrounder.pdf. Accessed December, 2014.

3. Malenfant EC, Lebel A, Martel L: Projections of the Diversity of the Canadian Population: 2006 to 2031. In Catalogue no. 91-551-X, Demography Division. Ottawa, Canada: Statistics Canada; 2010.

4. Newbold KB: Linking immigrant settlement, health, housing, and homelessness in Canada. Can Issues 2010, 24:28 31

5. Murdie R: Precarious Beginnings: The Housing Situation of Canadas Refugees. Montral: Association for Canadian Studies: Canadian Issues/Thmes Canadiens (Newcomers Experiences of Housing and Homelessness in Canada); 2010:47 53.

6. Colour of Poverty: Understanding the racialization of poverty in Ontario: An introduction in 2007. 2007; http://www.learningandviolence.net/ Irnteach/material/PovertyFactSheets-aug07.pdf.

7. Childrens Aid Society of Toronto: Greater trouble in greater Toronto: Child poverty in the GTA. 2008; http://www.torontocas.ca/wpcontent/uploads/ 2008/12/castchildpovertyreportdec2008.pdf. Accessed December, 2014.

8. Frankish CJ, Hwang SW, Quantz D: Homelessness and health in Canada. Can J Public Health 2005, 96(Supplement 2):S23 S28.

9. Hwang SW: Homelessness and health. Can Med Assoc J 2001, 164(2):229 233.

10. Perreault S: Visible Minorities and Victimization, 2004. In Canadian Centre for Justice Statistic Profile Series. Ottawa, Canada: Statistics Canada; 2008.

11. DeMaio FG, Kemp E: The deterioration of health status among immigrants to Canada. Glob Public Health 2010, 5(5):462 478.

12. Pascoe E, Richman L: Perceived discrimination and health: a meta-analytic review. Psychol Bull 2009, 135(4):531 554. 
13. Paradies $Y$ : A systematic review of empirical research on self-reported racism and health. Int J Epidemiol 2006, 35:888 901.

14. Williams DR, Neighbors HW, Jackson JS: Racial/ethnic discrimination and health: findings from community studies. Am J Public Health 2008, 98:\$29 \$37

15. Phelan J, Link BG, Moore RE, Stueve A: The stigma of homelessness: the impact of the label homeless on attitudes toward poor persons. Soc Psychol Q 1997, 60(4):323 337

16. Farrelly S, Clement S, Gabbidon J, Jeffery D, Dockery L, Lassman F, Brohan E, Henderson RC, Williams P, Howard LM, Thornicroft G: Anticipated and experienced discrimination amongst people with schizophrnia, bipolar disorder and major depressive disorder: a cross sectional study. BMC Psychiatry 2014, 14:157.

17. Ayalon L, Gum AM: The relationships between major lifetime discrimination, everyday discrimination, and mental health in three racial and ethnic groups of older adults. Aging Ment Health 2011, 15(5):587 594

18. Alvidrez J, Snowden LR, Kaiser DM: The experience of stigma among Black mental health consumers. J Health Care Poor Underserved 2008 19(3):874 893

19. Bhui K, Shanahan L, Harding G: Homelessness and mental illness: a literature review and a qualitative study of perceptions of the adequacy of care. Int J Soc Psychiatry 2006, 52(2):152 165.

20. Corrigan P: How stigma interferes with mental health care. Am Psychol 2004, 59(7):614 625.

21. Ahern J, Stuber J, Sandro G: Stigma, discrimination and the health of illicit drug users. Drug Alcohol Depend 2007, 88:188 196.

22. Bogart LM, Wanger GJ, Galvan FH, Landrine H, Klein DJ, Sticklor LA: Perceived discrimination and mental health symptoms among Black men with HIV. Cult Divers Ethn Minor Psychol 2011, 17:295 302.

23. Hausmann LRM, Jeong K, Bost JE, Ibrahim SA: Perceived discriminatino in health care and health status in a racially diverse sample. Med Care 2008, 46(9):905 914

24. Hsu L, Alden LE: Cultural influences on willingness to seek treatment for social anxiety in Chinese- and European-heritage students. Cult Divers Ethn Minor Psychol 2008, 14(3):215 223.

25. Williams DR, Mohammed SA: Discrimination and racial disparities in health: evidence and needed research. J Behav Med 2009 , 32(1):20 47

26. Dinos S, Stevens S, Serfaty M, Weich S, King M: Stigma: the feelings and experiences of 46 people with mental illness. Qualitative study. Br J Psychiatry 2004, 184:176 181

27. Gilvarry CM, Walsh E, Samele C, Hutchinson G, Mallett R, Rabe-Hesketh S, Fahy T, Van Os J, Murray RM: Life events, ethnicity and perceptions of discrimination in patients with severe mental illness. Soc Psychiatry Psychiatr Epidemiol 1999, 34(11):600 608

28. Gajardo C: A road to home: working with homeless immigrants and refugees. Can Issues 2010, 24:104 105

29. Bahm A, Forchuk C: Interlocking oppressions: the effect of a comorbid physical disability on perceived stigma and discrimination among mental health consumers in Canada. Health Soc Care Community 2009, 17(1):36 70

30. Corrigan $P$, Thompson VL, Lambert D, Sangster $Y$, Noel JG, Campbell J: Perceptions of discrimination among persons with serious mental illness. Psychiatr Serv 2003, 54(8):1105 1110.

31. Gary FA: Stigma: barrier to mental health care among ethnic minorities. Issues Ment Health Nurs 2005, 26:979 999.

32. Gee GC: A multilevel analysis of the relationship between institutional and individual racial discrimination and health status. Am J Public Health 2002, 92(4):615 623

33. Stuber J, Galea S, Ahern J, Blaney S, Fuller C: The association between multiple domains of discrimination and self-assessed health: a multilevel analysis of Latinos and blacks in four low-income New York City neighborhoods. Health Serv Res 2003, 38:1735 1759.

34. Janssen I, Hanssen M, Bak R, Bijl RV, DeGraaf R, Vollebergh W, McKenzie K, Van Os J: Discrimination and delusional ideation. Br J Psychiatry 2003, 182(1):71 76 .

35. Haan M: Does immigrant residential crowding reflect hidden homelessness? Can Stud Popul 2011, 38(1 2):43 59.

36. Enns R: Immigrant households and homelessness. Can Issues 2005, 127:130.
37. Chiu S, Redelmeier DA, Tolomiczenko G, Kiss A, Hwang S: The health of homeless immigrants. J Epidemiol Community Health 2009, 63(11):943 948

38. Dealberto M-JCC, Middleboro A, Farrell S: Symptoms of schizophrenia and psychosis according to foreign birth in a Canadian sample of homeless persons. Psychiatr Serv 2011, 62(10):1187 1193

39. Meadows LM, Thurston WE, Melton C: Immigrant women s health. Soc Sci Med 2001, 52:1451 1458.

40. Li HZ, Browne AJ: Defining mental illness and accessing mental health services: Perspectives of Asian Canadians. Can J Commun Ment Health 2000, 19(1):143 160

41. Khanlou N, Koh JG, Mill C: Cultural identity and experiences of prejudice and discrimination of Afghan and Iranian immigrant youth. Int J Ment Heal Addict 2008, 6(4):494 513.

42. Benbow S, Forchuk C, Ray SL: Mothers with mental illness experiencing homelessness: a critical analysis. J Psychiatr Ment Health Nurs 2011, 18(8):687 695

43. Paradis E, Novac S, Sarty M, Hulchanski JD: Homelessness and Housing among Status Immigrant, Non-Status Migrant, and Canadian-Born Families in Toronto. Montral: Association for Canadian Studies: Canadian Issues/ Thmes Canadiens (Newcomers Experiences of Housing and Homelessness in Canada); 2010:36 39.

44. Simich $L$ : Refugee mental health and the meaning of home . Can Issues 2010, 24:68 72

45. Edge $\mathrm{S}$, Newbold B: Discrimination and the health of immigrants and refugees: Exploring Canada s evidence base and directions for future research in newcomer receiving countries. J Immigr Minor Health 2013, 15(1):141 148

46. Link BG, Yang LH, Phelan JC, Collins PY: Measuring mental illness stigma. Schizophr Bull 2004, 30(3):511 538.

47. Hwang S, Stergiopoulos V, O'Campo P, Gozdzik A: Ending homelessness among people with mental illness: The At Home/Chez Soi randomized trial of a Housing First intervention in Toronto. BMC Public Health 2012, 12:787.

48. Goering PN, Streiner DL, Adair C, Aubry T, Barker J, Distasio J, Hwang SW, Komaroff J, Latimer E, Somers J, Zabkiewicz DM: The At Home/Chez Soi trial protocol: a pragmatic, multi-site, randomised controlled trial of a Housing First intervention for homeless individuals with mental illness in five Canadian cities. BMJ Open 2011, 1(2):e000323.

49. Starks H, Trinidad SB: Choose your method: a comparison of phenomenology, discourse analysis, and grounded theory. Qual Health Res 2007, 17(10):1372 1380.

50. Charmaz K: Grounded Theory Approaches to Qualitative Research: A Reader on Theory and Practice. New York: Oxford University Press; 2004

51. Braun V, Clarke V: Using thematic analysis in psychology. Qual Res Psychol 2006, 3:77 101.

52. Skosireva A, O'Campo P, Zerger S, Chambers C, Gapka S, Stergiopoulos V: Perceived discrimination among homeless adults with mental illness in health care settings. BMC Health Serv Res 2014, 14:376

53. Minior T, Galea S, Stuber J, Ahern J, Ompad D: Racial differences in discrimination experiences and responses among minority substance users. Ethn Dis 2003, 13:521 527.

54. Sohler N, Li X, Cunningham C: Perceived discrimination among severely disadvantaged people with HIV infection. Public Health Rep 2007 122:347 355 .

55. Padgett D: There s no place like (a) home: ontological security among persons with serious mental illness in the United States. Soc Sci Med 2007, 64(9):1925 1936

56. Yanos PT, Stefancic A, Tsemberis S: Psychological community integration among people with psychiatric disabilities and nondisabled community members. J Community Psychol 2011, 39(4):390 401.

57. Thompson VLS, Noel JG, Campbell J: Stigmatization, discrimination, and mental health: The impact of multiple identity status. Am J Orthopsychiatry 2004, 74(4):529 544

58. Berg AO, Melle I, Rossberg Jl, Romm KL, Larsson S, Lagerberg TV, Andreassen OA, Hauff E: Perceived discrimination is associated with severity of positive and depression/anxiety symptoms in immigrants with psychosis: a cross-sectional study. BMC Psychiatry 2011, 11:77

59. Schomerus G, Schwahn C, Holzinger A, Corrigan PW, Grabe HJ, Carta MG, Angermeyer MC: Evolution of public attitudes about mental illness: a systematic review and meta-analysis. Acta Psychiatr Scand 2012, 125:440 452. 
60. Saechao F, Sharrock S, Reicherter D, Livingston J, Aylward A, Whisnant J, Koopman C, Kohli S: Stressors and barriers to using mental health services among diverse groups of first-generation immigrants to the United States. Community Ment Health J 2012, 48(1):98 106.

61. Cole ER: Intersectionality and research in psychology. Am Psychol 2009, 64(3):170 180 .

doi:10.1186/s12888-014-0353-1

Cite this article as: Zerger et al:: Differential experiences of discrimination among ethnoracially diverse persons experiencing mental illness and homelessness. BMC Psychiatry 2014 14:353.

\section{Submit your next manuscript to BioMed Central and take full advantage of:}

$\otimes$ Convenient online submission

$\otimes$ Thorough peer review

$\otimes$ No space constraints or color $\nabla$ gure charges

$\bigotimes$ Immediate publication on acceptance

Q Inclusion in PubMed, CAS, Scopus and Google Scholar

$\otimes$ Research which is freely available for redistribution 